\title{
Schuld, Verantwortung und Wahrheit
}

\author{
Dieter Dölling • Henning Saß
}

Online publiziert: 11. April 2015

(C) Springer-Verlag Berlin Heidelberg 2015

\section{Guilt, responsibility and truth}

Schuld, Verantwortung und Wahrheit sind Grundbegriffe der deutschen Strafrechtspflege. Nach dem Schuldprinzip darf nur bestraft werden, wer die mit Strafe bedrohte Handlung schuldhaft begangen hat. Mit der Bestrafung wird der schuldhaft handelnde Täter für die Tat zur Verantwortung gezogen. Die Verwirklichung des Schuldprinzips setzt voraus, dass im Strafverfahren der wahre Sachverhalt ermittelt wird (Bundesverfassungsgericht, NJW 2013, S. 1058, 1060). Schuld, Verantwortung und Wahrheit sind freilich sehr komplexe Konzepte, deren Inhalt und Berechtigung umstritten sind. Ihre Verwirklichung in der Praxis stellt soweit sie überhaupt möglich ist - hohe Anforderungen an die im Strafverfahren Tätigen. Mit Fragen aus diesem Problemkreis befassen sich die Beiträge des vorliegenden Hefts der Zeitschrift Forensische Psychiatrie, Psychologie, Kriminologie.

Die Arbeit von Nenad Vasic et al. behandelt die historische Entwicklung des Schuldbegriffs. Es wird gezeigt, wie sich die Vorstellungen über die Verantwortlichkeit des Menschen für seine Handlungen zunehmend verfeinert haben und dass diese Vorstellungen durch theologische und philo-

Prof. Dr. jur. D. Dölling $(\varangle)$

Institut für Kriminologie, Universität Heidelberg,

Friedrich-Ebert-Anlage 6-10,

69117 Heidelberg, Deutschland

E-Mail: doelling@krimi.uni-heidelberg.de

Prof. Dr. med. H. Saß

Klinik für Psychiatrie, Psychotherapie und Psychosomatik,

Universitätsklinikum Aachen,

Pauwelsstr. 30,

52704 Aachen, Deutschland

E-Mail: hsass@ukaachen.de sophische Konzepte beeinflusst werden. - Die Frage, ob es eine Verantwortung für die Entwicklung der eigenen Persönlichkeit gibt, erörtert der Beitrag von Henning $S a \beta$. Die Begriffe der Persönlichkeit und der Persönlichkeitsstörung werden definiert und unter Bezugnahme auf die Fähigkeit des Menschen, sich zu sich selbst zu verhalten und seine Lebensführung mitzugestalten, wird eine Mitverantwortlichkeit des Menschen für seine Persönlichkeitsentwicklung angenommen, was auch eine Voraussetzung für psychotherapeutische Veränderungsbemühungen darstellt.

Mit der Problematik der Wahrheitsfindung durch die Strafgerichte befasst sich der Beitrag von Andreas Mosbacher. Er stellt dar, wie die Wahrheitsfindung nach den Regeln der Strafprozessordnung über die Beweiserhebung und die Beweiswürdigung zu erfolgen hat, zeigt Quellen von Fehlurteilen auf und erörtert Möglichkeiten, das Risiko von Fehlurteilen zu reduzieren. - Der Angeklagte hat das Recht, sich zur Anklage zu äußern oder zu schweigen. Alexander Ignor erörtert die Frage, zu welchem Verhalten der Verteidiger dem Angeklagten raten soll. Er legt dar, welche prozessuale Bedeutung der Einlassung und dem Schweigen des Angeklagten zukommt und in welchen Konstellationen es sich für den Angeklagten empfiehlt zu schweigen, den Tatvorwurf zu bestreiten oder ein Geständnis abzulegen. Mit dem Schweigen des Angeklagten setzt sich Hans-Ludwig Kröber aus der Sicht eines Psychiaters auseinander. Er wirft die Frage auf, ob es dem Angeklagten stets hilft, wenn der Verteidiger ihn zum Schweigen veranlasst, und zeigt, dass dann, wenn der Tatnachweis mit hoher Wahrscheinlichkeit gelingen wird, ein Geständnis für den Angeklagten die Chance eröffnen kann, sich konstruktiv mit der Tat auseinanderzusetzen und erste Schritte auf dem Weg in die soziale Reintegration zu gehen. Erörtert wird auch, wie sich der forensische Psychiater gegenüber einem Beschuldigten, der ein Geständnis erwägt, verhalten soll. 
Schließlich enthält dieses Heft einen Beitrag von $\mathrm{Car}$ los Canela et al. über die invalidenversicherungsrechtliche Begutachtung in der Schweiz. Leistungen der Invalidenversicherung der Schweiz setzen eine Beeinträchtigung der körperlichen, geistigen oder psychischen Gesundheit voraus, die eine vollständige oder teilweise Erwerbsunfähigkeit verursacht. Die Autoren erörtern insbesondere die schweizerische Rechtsprechung, wonach anhaltende somatoforme Schmerzstörungen und pathogenetisch-ätiologisch unklare syndromale Beschwerdebilder ohne nachweisbare organische Grundlage in der Regel durch eine zumutbare Willensanstrengung überwunden werden können und daher keine Invalidität begründen. Auch in diesem Zusammenhang spielt also die Verantwortungszuschreibung eine Rolle.

Interessenkonflikt D. Dölling und H. Saß geben an, dass kein Intressenkonflikt besteht. 